\title{
Dual Statistical Systems and Geometrical String
}

\author{
George K. Savvidy \\ Physics Department, University of Crete \\ 71409 Iraklion, Crete, Greece \\ and \\ Yerevan Physics Institute, 375036 Yerevan, Armenia \\ Konstantin G.Savvidy \\ Princeton University, Department of Physics \\ P.O.Box 708, Princeton, New Jersey 08544 \\ Paul G.Savvidy \\ Physics Department, University of Crete \\ 71409 Iraklion, Crete, Greece
}

\begin{abstract}
We analyse statistical system with interface energy proportional to the length of the edges of interface. We have found the dual system high temperature expansion of which equally well generates surfaces with linear amplitude. These dual systems are in the same relation as 3D Ising ferromagnet to the 3D Gauge spin system.
\end{abstract}


1. Low temperature expansion of the Ising ferromagnet has a beautiful geometrical representation of the partition function in terms of the sum over the paths on the two-dimensional lattice and over the surfaces on three-dimensional lattice. The corresponding amplitudes are proportional to the length of the paths and to the area of the random surfaces. This geometrical representation allows to show, that high temperature expansion of 2D Ising ferromagnet is dual to that of low temperature expansion and that the system is therefore self-dual [1, 2, 3, 4, 5, [0]. In the case of 3D Ising ferromagnet

$$
H_{\text {Ising }}^{3 D}=-\sum_{\text {links }} \sigma \sigma
$$

the high temperature expansion does not coincide with its low temperature expansion and can be represented as a sum over the random walks with the amplitude which is proportional to the length of the paths.

In 1971 Franz Wegner succeeded to construct the spin system with local interaction low temperature expansion of which coincides with the random walks in 3D with the length amplitude and with high temperature expansion of which coincides with the sum over the random surfaces with the area amplitude. In this natural way he have found the Gauge spin Hamiltonian which is dual to 3D Ising ferromagnet [6]

$$
H_{\text {Gauge }}^{3 D}=-\sum_{\text {plaquettes }} \sigma \sigma \sigma \sigma .
$$

One motivation for the study of the spin systems (1) is that they can help to understand the dynamics of random surfaces with area action [7, 8, 9, 10, 11, 12, 13

In the recent articles [14, 15] the authors formulated a new class of statistical systems, whose interface energy is associated with the edges of the interface. These systems again have geometrical representation of the low temperature partition function in terms of the sum over paths and surfaces, but with amplitude which is proportional to total curvature of the path on the two-dimensional lattice and to the linear size of the surface in three dimensions [16, 17, 18, 19, 20, 21].

In terms of Ising spin variables $\sigma_{\vec{r}}$ the Hamiltonian of this system has the form [14, 15]

$$
H_{\text {Gonihedric }}^{3 D}=-\sum_{\vec{r}, \vec{\alpha}, \vec{\beta}} \sigma_{\vec{r}} \sigma_{\vec{r}+\vec{\alpha}} \sigma_{\vec{r}+\vec{\alpha}+\vec{\beta}} \sigma_{\vec{r}+\vec{\beta}}
$$

where $\vec{r}$ is a three-dimensional vector on the lattice $Z^{3}$ whose components are integer and $\vec{\alpha}, \vec{\beta}$ are unit vectors parallel to axis. Together with usual symmetry group $Z_{2}$ the system (2) has an extra symmetry: one can independently flip the spins on any combination of planes (spin layers). We should stress that the Ising spins in (2) are on the vertices of the lattice $Z^{3}$ and are not on the links, as it is in (1b).

It is an important fact that there exist dual representation of the random surfaces on the lattice with area law in terms of Ising and Wegner Hamiltonians (1a), (1b) [22, 23, 24, 25, 26]. Our aim is to construct Hamiltonian which is dual to $H_{\text {Gonihedric }}^{3 D}$ (2) and equally well describes surfaces with linear amplitude and to compare these complementary systems with each other. 
2. The partition function of the system (2) can be written in the form

$$
Z(\beta)=\sum_{\{\sigma\}} \exp \left\{-\beta H_{\text {Gonihedric }}^{3 D}\right\}
$$

and as it was shown in 14, 15 the low temperature expansion is extended over all closed surfaces $\{M\}$ with the restriction that only an even number of plaquettes can intersect at any given edge $(2 r=0,2,4$. $)$ and that only one plaquette is at every given place

$$
Z(\beta)=\sum_{\{M\}} \exp \{-2 \beta A(M)\},
$$

where $A(M)$ is linear-gonihedric action [16, 17, 18] which is equal to

$$
A(M)=\sum_{<i, j>} \lambda_{i, j} \cdot\left|\pi-\alpha_{i, j}\right|,
$$

the summation is over all edges $\langle i, j\rangle$ of $M, \alpha_{i, j}$ is the angle between two neighbour plaquettes of $M$ in $Z^{3}$ having a common edge $\langle i, j\rangle$ of the length $\lambda_{i, j}$. As it is easy to see $A(M)$ is proportional to the linear size of the surface $M$ [16, 17, 18.

The Hamiltonian (2) and the partition function (3) provides the representation (4) of the randomly fluctuating surfaces on the lattice with gonihedric action $A(M)$ in terms of locally interacting fields-spins [14, 15]. To construct the dual Hamiltonian we should find the geometrical representation of the high temperature expansion of the system (2),(3). Let us consider for that high temperature expansion of (3)

$$
Z(\beta)=\sum_{\{\sigma\}} \prod_{\text {plaquetts }} \operatorname{ch} \beta \cdot\{1+\operatorname{th} \beta \cdot(\sigma \sigma \sigma \sigma)\} .
$$

Opening the brackets and summing over $\sigma$ one can see that only those terms produce nonzero contribution which contain an even number of plaquettes on every given vertex, therefore

$$
Z(\beta)=(2 \operatorname{ch} \beta)^{3 N^{3}} \sum_{\{\Sigma\}}(\operatorname{th} \beta)^{s(\Sigma)},
$$

where the summation is extended over all surfaces $\{\Sigma\}$ with an even number of plaquettes at any given vertex. The $s(\Sigma)$ is the number of plaquettes of $\Sigma$, e.g. the area of the surface. Open surfaces are allowed. In the next section we will describe in details this set of surfaces $\{\Sigma\}$ and will introduce the concept of the group structure on this set.

3. As we have seen, the high temperature expansion (6) of the gonihedric system (2), (3) in $3 \mathrm{D}$ is extended over surfaces $\{\Sigma\}$ which can be considered as a collection of plaquettes on a cubic lattice with the restriction that only an even number of plaquettes can intersect at any given vertex and that only one plaquette is at every given place.

Let us attach plaquette variables $U_{P}$ to each plaquette $P$ of $Z^{3}$ 


$$
U_{P}=-1 \quad \text { if } P \in M \quad \text { and } \quad U_{P}=1 \text { if } \notin M
$$

There are twelve plaquettes $P$ incident to every vertex of the lattice. The constraint on the plaquette variables $U_{P}$ in every vertex

$$
\prod_{12 \text { plaquettes incident to vertex }} U_{P}=1,
$$

uniquely characterizes our set of surfaces $\{\Sigma\}$ in (6).

Now one can introduce the group structure on this set of surfaces $\{\Sigma\}(8)$. Let us consider two surfaces $\Sigma^{1}$ and $\Sigma^{2}$ and denote their plaquette variables as $U_{P}^{1}$ and $U_{P}^{2}$ respectively. Let us define the group product of those two surfaces as

$$
U_{P}=U_{P}^{1} \cdot U_{P}^{2}
$$

According to this definition a given plaquette belongs to a group product of two surfaces $\Sigma=\Sigma^{1} \otimes \Sigma^{2}$ only if it belongs exactly to one of $\Sigma^{1}$ or $\Sigma^{2}$.

One should check that the group product defined in this way leaves the surfaces in the same class (8). Indeed if (8) holds for $\Sigma^{1}$ and $\Sigma^{2}$ then it holds for the surface product $\Sigma$, that is $\Sigma$ also have even number of plaquettes on its every vertex (8). The inverse element of $\Sigma$ coincides with itself. The set of surfaces $\{\Sigma\}$ (8) finally forms an Abelian group $G$.

One can show that the whole group $G$ is a product of the local group $G_{\xi}$. This group $G_{\xi}$ has four elements - elementary "matchbox" surfaces,

$$
e=\quad, g_{\chi}=\quad, g_{\eta}=\quad, g_{\varsigma}=
$$

with the multiplication table

$$
\begin{gathered}
e \cdot g_{\chi, \eta, \varsigma}=g_{\chi, \eta, \varsigma}, \\
g_{\chi} \cdot g_{\chi}=g_{\eta} \cdot g_{\eta}=g_{\varsigma} \cdot g_{\varsigma}=e \\
g_{\chi} \cdot g_{\eta}=g_{\varsigma},
\end{gathered}
$$

which follows from the multiplication law (9).

With the help of $G_{\xi}$ one can reconstruct any surface $\Sigma$ of the set $\{\Sigma\}(8)$. Indeed any set of elementary matchbox surfaces $e, g_{\chi}, g_{\eta}, g_{\varsigma}(10),(11)$ distributed independently over the lattice $Z^{3}$ describes some allowed surface $\Sigma$ and any given surface from $\{\Sigma\}(8)$ can be decomposed into the product of $G_{\xi}$

$$
\Sigma=\prod_{\xi} \cdot G_{\xi}
$$

This approach allows to describe the original surface $\Sigma$ in terms of a new independent matchbox spin variable

$$
G_{\xi}=\left\{e(\xi), g_{\chi}(\xi), g_{\eta}(\xi), g_{\varsigma}(\xi)\right\}
$$

which should be attached to the centers of the cubes $\xi$ of the original lattice $Z^{3}$ or to vertices of the dual lattice $Z^{\star}$. 
This is what we aimed at: to express the surface configuration $\Sigma$ with the constraints (8) in terms of independent local variable $G_{\xi}$. The group $G_{\xi}$ is an Abelian group of the fourth order and therefore has four one-dimensional irreducible representations

$$
\left.E=\{1,1,1,1\}, R^{\chi}=\{1,1,-1,-1\}\right), R^{\eta}=\{1,-1,1,-1\}, R^{\varsigma}=\{1,-1,-1,1\}
$$

with the orthogonality relations

$$
\begin{gathered}
\sum_{G_{\xi}} R^{l}\left(G_{\xi}\right) \cdot R^{m}\left(G_{\xi}\right)=4 \delta^{l, m}(l, m=\chi, \eta, \varsigma) \\
\sum_{G_{\xi}} R^{\chi}\left(G_{\xi}\right) R^{\eta}\left(G_{\xi}\right) R^{\varsigma}\left(G_{\xi}\right)=4
\end{gathered}
$$

We will use this representations to express algebraically the matchbox spin variable $G_{\xi}$. The next step is to express the amplitude of $\Sigma$ in terms of this independent variables and to construct the dual Hamiltonian.

4. In the high temperature expansion (6), the energy of the surface $\Sigma$ is equal to the number of plaquets $s(\Sigma)$, that is to area. We would like to construct now a new system of locally interacting matchbox spins $G_{\xi}$ with identical low temperature expansion. For that we should properly organize local interaction of the matchbox spins $G_{\xi}$.

The dual Hamiltonian is nonhomogeneous in the directions $\chi, \eta$, and $\varsigma$ and is equal to

$$
H_{\text {dual }}=\sum_{\xi} H_{\xi, \xi+\chi}+H_{\xi, \xi+\eta}+H_{\xi, \xi+\varsigma},
$$

where $\chi, \eta$, and $\varsigma$ are unit vectors in the corresponding directions of the dual lattice and

$$
\begin{aligned}
H_{\xi, \xi+\chi} & \equiv H\left(G_{\xi}, G_{\xi+\chi}\right)=-R^{\chi}(\xi) \cdot R^{\chi}(\xi+\chi), \\
H_{\xi, \xi+\eta} & \equiv H\left(G_{\xi}, G_{\xi+\eta}\right)=-R^{\eta}(\xi) \cdot R^{\eta}(\xi+\eta), \\
H_{\xi, \xi+\varsigma} & \equiv H\left(G_{\xi}, G_{\xi+\varsigma}\right)=-R^{\varsigma}(\xi) \cdot R^{\varsigma}(\xi+\varsigma) .
\end{aligned}
$$

The partition function of the dual system (14), (15) can be written in the form

$$
Z\left(\beta^{\star}\right)=\sum_{\left\{G_{\xi}\right\}} \exp \left\{-\beta^{\star} H_{\text {dual }}\right\} .
$$

Now we should check, that the low temperature expansion of the dual system (16) indeed coincides with the high temperature expansion of the original system (6). As we will see this indeed takes place and we can expect that high temperature expansion of the dual Hamiltonian will provide us with the new lattice representation of the random surfaces with gonihedric action. In the next section we will show that two systems (2) and (14), (15) are indeed dual to each other. 
5. Let us define the surface of interface $\Sigma$ for the matchbox spins $G_{\xi}$ in the following way: plaquette $P$ belongs to $\Sigma$ if the product of neighboring matchbox spins is equal to -1

$$
P_{\xi, \xi+\chi} \in \Sigma \quad \text { if } \quad R^{\chi}\left(G_{\xi}\right) \cdot R^{\chi}\left(G_{\xi+\chi}\right)=-1
$$

and

$$
P_{\xi, \xi+\chi} \notin \Sigma \quad \text { if } \quad R^{\chi}\left(G_{\xi}\right) \cdot R^{\chi}\left(G_{\xi+\chi}\right)=1 .
$$

In the same way we should define $P_{\xi, \xi+\eta}$ and $P_{\xi, \xi+\varsigma}$. These surfaces are of the class $\{\Sigma\}$ (8) because plaquette variables $U_{P}$ defined as

$$
\begin{aligned}
U_{\xi, \xi+\chi} & =R^{\chi}\left(G_{\xi}\right) \cdot R^{\chi}\left(G_{\xi+\chi}\right) \\
U_{\xi, \xi+\eta} & =R^{\eta}\left(G_{\xi}\right) \cdot R^{\eta}\left(G_{\xi+\eta}\right) \\
U_{\xi, \xi+\varsigma} & =R^{\varsigma}\left(G_{\xi}\right) \cdot R^{\varsigma}\left(G_{\xi+\varsigma}\right)
\end{aligned}
$$

identically resolves the constranes (8). The correspondence between matchbox spin configurations and surfaces $\Sigma$ is four-to-one, instead of two-to-one in the case of surfaces of interface of the Ising ferromagnet.

By construction the energy of the surface $\Sigma$ of matchbox spin interface is equal to its area and therefore the low temperature expansion of the dual system (16) indeed coincides with the high temperature expansion of the original system (6).

6. As the last step in this construction we should prove that the high temperature expansion of the dual system (14), (15) is equivalent to the sum over random surfaces with the amplitude which is proportional to the linear size of the surface $A(M)$ and coincides with the low temperature expansion (4) of (3).

Expanding the partition function (16) for small $\beta^{\star}$

$$
Z\left(\beta^{\star}\right)=\sum_{\left\{G_{\xi}\right\}} \prod_{\xi}\left(c h \beta^{\star}\right)^{3}\left(1-t h \beta^{\star} \cdot H_{\xi, \xi+\chi}\right)\left(1-t h \beta^{\star} \cdot H_{\xi, \xi+\eta}\right)\left(1-t h \beta^{\star} \cdot H_{\xi, \xi+\varsigma}\right)
$$

and opening the brackets we will have the terms of the form

$$
\sum_{\left\{G_{\xi}\right\} \text { over paths on } Z^{\star 3}} R(\xi)
$$

representing the chains of $R^{\prime} s$ (13) of different lengths on the lattice $Z^{\star 3}$. Let us see which of those terms are nonzero. Using relations

$$
\begin{gathered}
R^{\chi}\left(G_{\xi}\right) R^{\chi}\left(G_{\xi}\right)=R^{\eta}\left(G_{\xi}\right) R^{\eta}\left(G_{\xi}\right)=R^{\varsigma}\left(G_{\xi}\right) R^{\varsigma}\left(G_{\xi}\right)=1 \\
R^{\chi}\left(G_{\xi}\right) R^{\eta}\left(G_{\xi}\right) R^{\varsigma}\left(G_{\xi}\right)=1
\end{gathered}
$$

which hold for the irreducible representations (13), one can get

$$
\sum_{G_{\xi}} H_{\xi-\chi, \xi} H_{\xi, \xi+\chi}=4 R^{\chi}(\xi-\chi) \cdot R^{\chi}(\xi+\chi),
$$




$$
\sum_{G_{\xi}} H_{\xi-\chi, \xi} H_{\xi, \xi+\eta}=\sum_{G_{\xi}} H_{\xi-\chi, \xi+\varsigma}=0 .
$$

Last two relations tell us that any loop which contains one turn at the right angle is equal to zero. We have three extra nonzero elementary verteces

$$
\begin{gathered}
\sum_{G_{\xi}} H_{\xi, \xi+\chi} H_{\xi, \xi+\eta} H_{\xi, \xi+\varsigma}=-4 R^{\chi}(\xi+\chi) R^{\eta}(\xi+\eta) R^{\varsigma}(\xi+\varsigma) \\
\sum_{G_{\xi}} H_{\xi-\chi, \xi} H_{\xi, \xi+\chi} H_{\xi-\eta, \xi} H_{\xi, \xi+\eta}=4 R^{\chi}(\xi-\chi) R^{\chi}(\xi+\chi) R^{\eta}(\xi-\eta) R^{\eta}(\xi+\eta) \\
\sum_{G_{\xi}} H_{\xi-\chi, \xi} H_{\xi, \xi+\chi} H_{\xi-\eta, \xi} H_{\xi, \xi+\eta} H_{\xi-\varsigma, \xi} H_{\xi, \xi+\varsigma}= \\
4 R^{\chi}(\xi-\chi) R^{\chi}(\xi+\chi) R^{\eta}(\xi-\eta) R^{\eta}(\xi+\eta) R^{\varsigma}(\xi-\varsigma) R^{\varsigma}(\xi+\varsigma)
\end{gathered}
$$

and all other verteces are equal to zero.

So, high temperature expansion of the dual system (14), (15) contains only those loops on the lattice $Z^{\star 3}$ which have only one of those four nonzero verteces of the type (23) and (24). One can imagine every term of this expansion as a "skeleton" constructed by the loops of "bones" restricted by the constraints (23) and (24). The amplitude is proportional to the total length of this bones. The partition function therefore have the form

$$
Z\left(\beta^{\star}\right)=\sum_{\text {skeletons }}\left(\text { th } \beta^{\star}\right)^{A(\text { skeleton })}
$$

where $A$ (skeleton) is the total length of the bones.

Nontrivial fact consists in the statement that one can "dress up" this skeletons by plaquettes so that bones will appear as the right angle edges of the surface $M$. In that case the $A$ (skeleton) becomes identically equal to $A(M)$ and summation over skeletons reduce to the summation over surfaces $M$ with linear-gonihedric action $A(M)$. This identification is possible only because bones can join together and form the loops only though the vertices (23) and (24). So we have

$$
\ln \left(t h \beta^{\star}\right)=-2 \beta
$$

and both systems (2) and (13),(14) and (15) are dual to each other in the same way as 3D Ising ferromagnet (1a) is dual to 3D Wegner gauge Hamiltonian (1b). They are complementary to each other in the sense that (1b) describe random surfaces with area law and (14),(15) with linear-gonihedric law.

Here raises the question of whether or not the system exhibit a phase transition. Because the energy functional is proportional to the linear size of the surfaces one can expect that the system will show phase transition in 3D which should be of the same nature as it is in the case of 2D Ising ferromagnet.

We gratefully acknowledge stimulating discussions with Franz Wegner. It is a pleasure to thank J.Ambjorn, D.Gross, B.Durhuus, E.Marinari, H.Nielsen, E.Paschos, E.Floratos, R.Flume, R.Schneider for helpful conversations.

This work was sponsored in part by the Danish Natural Science Research Council. 


\section{References}

[1] B.L.van der Waerden. Z.Physik 118 (1941) 473

[2] H.A.Kramers and G.H.Wannier. Phys.Rev. 60 (1941) 252

[3] E.Ising. Z.Physik 31 (1925) 253

[4] L.Onsager. Phys.Rev. 65 (1944) 117

[5] M.Kac and J.C.Ward. Phys.Rev. 88 (1952) 1332

[6] F.J. Wegner, J. Math. Phys.12 (1971) 2259

[7] V.S.Dotsenko and et.all. Phys.Rev.Lett. 71 (1993) 811

[8] B.Durhuus, J.Frohlich and T.Jonsson, Nucl.Phys. B240 (1984) 453

[9] J.Ambjorn, B.Durhuus, J.Frohlich and P.Orland, Nucl.Phys. B270 (1986) 457

[10] E.Fradkin, M.Srednicky and L.Susskind. Phys.Rev. D21 (1980) 2885

[11] C.Itzykson. Nucl.Phys. B210 (1982) 477

[12] A.Casher, D.Foerster and P.Windey. Nucl.Phys. B251 (1985) 29

[13] A.Polyakov. Gauge fields and String. (Harwood Academic Publishers, 1987)

[14] G.K.Savvidy and F.J.Wegner. Nucl.Phys.B413(1994)605.

[15] G.K.Savvidy and K.G.Savvidy. Phys.Lett. B324 (1994) 72

[16] R.V. Ambartzumian, G.K. Savvidy, K.G. Savvidy and G.S. Sukiasian. Phys. Lett. B275 (1992) 99

[17] G.K. Savvidy and K.G. Savvidy. Int. J. Mod. Phys. A8 (1993) 3993

[18] G.K. Savvidy, K.G. Savvidy. Mod.Phys.Lett. A8 (1993) 2963

[19] G.K.Savvidy and R.Schneider. Comm. Math. Phys. 161 (1994) 283

[20] B.Durhuus and T.Jonsson. Phys.Lett. B297 (1992) 271

[21] C.F.Baillie and D.A.Johnston. Phys.Rev D 45 (1992) 3326

[22] H.B.Nielsen and P.Olesen. Phys.Lett. B32 (1970) 203

[23] A.Billoire, D.J.Gross and E.Marinari. Phys.Lett. B139 (1984) 75

[24] V.A.Kazakov. Phys.Lett. B150 (1985) 282

[25] J.Ambjorn, D.Durhuus and J.Frohlich. Nucl.Phys. B257 (1985) 433

[26] F.David. Nucl.Phys. B257 (1985) 543 